\title{
Editorial
}

\section{Emerging attosecond technologies}

\author{
Guest editors \\ Johan Mauritsson $^{1}$ (D), \\ Giulio Vampa ${ }^{2}$ (iD and \\ Caterina Vozzi ${ }^{3}$ (i) \\ ${ }^{I}$ Department of Physics, Lund \\ University, Lund, Sweden \\ ${ }^{2}$ Stanford PULSE Institute, \\ SLAC National Accelerator \\ Laboratory, Menlo Park, CA \\ 94025, United States of America \\ ${ }^{3}$ Institute for Photonics and \\ Nanotechnologies, CNR-IFN, \\ 20133 Milano, Italy \\ E-mail: johan.mauritsson@fysik. \\ lth.se,gvampa@stanford.edu \\ and caterina.vozzi@cnr.it
}

\begin{abstract}
The quest for fundamental scientific knowledge triggers technological advancement. This has been particularly true for attosecond science in recent years: we assisted in the development of many new approaches — both experimentally and theoretically — that will have a high impact, not only on atomic and molecular physics, but also on optical technology. This special issue collects several of these contributions.
\end{abstract}

Attosecond science [1] studies the motion of electrons on their natural timescale, which is typically of the order of attoseconds $\left(1 \mathrm{as}=10^{-18} \mathrm{~s}\right)$. Since the first demonstration in 2001 [2, 3], this research field has grown exponentially and it has captured the attention of the scientific community with the exciting promise of deepening our understanding of fundamental processes occurring in atoms and molecules. This field has already provided a number of seminal experiments, including the measurement of Auger decay [4], autoionization [5], time-delay in photoionization [6, 7], electron re-collision [8, 9], electronic superpositions [10] and charge migration in molecules [11] among others.

Attosecond science relies on the generation of attosecond pulses through the process of high-order harmonic generation (HHG). High-order harmonics are generated due to the strong nonlinear interaction when an intense laser pulse is focused in a gas medium and very high odd-order harmonics of the driving pulse frequency can be generated $[12,13]$. This nonlinear interaction leads to the production of a train of light bursts in the extreme ultra-violet (XUV) spectral region with attosecond duration [14]. Commercial table-top HHG sources nowadays generate a coherent flux of XUV photons comparable to synchrotron sources with higher peak brightness. The pulses in the attosecond pulse train generated through HHG are separated by half of the period of the driving electric field. For probing electron dynamics, it is often necessary to isolate a single pulse in the train and synchronize it with another optical pulse. This is done by isolating a single emission event for the driving pulse in the HHG process. Different schemes have been demonstrated for gating the HHG process, i.e. isolating a single ionization event, in order to achieve the generation of isolated attosecond pulses. The selection of one emission event can be achieved in different ways, notably by spectral [15] or temporal gating [16, 17].

Until a decade ago, Ti:sapphire lasers were the only sources available for the exploration of molecular dynamics in the few-femtosecond and attosecond regime, because of their unsurpassed amplification bandwidth, which allowed intense pulse compression down to the single-cycle domain [18]. Because both the maximum photon energy in the harmonic spectrum and the spectral bandwidth of the attosecond pulse increase as the square of the driving field wavelength, longer driving wavelengths extend the HHG cutoff up to the water window $(300-500 \mathrm{eV})$ and beyond [19-21]. State of the art isolated attosecond pulses as short as 50 as have been generated in this spectral range.

Due to the increase of knowledge that attosecond science may provide in fundamental molecular processes, it is also one of the strategic areas of investments by the European Community. The Extreme Light Infrastructure is a European Strategy Forum on Research Infrastructures ESFRI project for the 
investigation of light-matter interactions at highest intensities and shortest time scales and the Hungarian pillar is completely devoted to attosecond science [22].

Recently, several exciting new routes have been explored in attosecond science. The study of chiral molecules has been allowed by the manipulation of HHG polarization and the generation of circularly polarized harmonics [23, 24]. Attosecond pulse trains and isolated attosecond pulses have allowed transient absorption and reflection measurements with extreme temporal resolution. In this framework, one of the 'hot topics' is indeed the study of electron dynamics in solids, such as the real-time study of electron transfer from valence to conduction band in semiconductors $[25,26]$, the electron and hole dynamics in germanium [27] and the core-exciton dynamics in $\mathrm{SiO}_{2}$ [28].

Experiments on solids are complemented by another emerging hot-topic regarding the interaction of intense fields with crystals. In recent years, many strong-field phenomena occurring in gases have indeed been observed to occur in solids too, such as strong-field excitations leading to field-controlled ultrafast currents [29] and the generation of high-order harmonics [30, 31].

Last but not least, the demonstration of very short pulses down to the attosecond regime is expected to happen at free electron lasers (FELs) soon, following the demonstrated operation of FELs in the single-spike mode [32].

All these exciting advances in attosecond science would not have been possible without cutting edge technological developments in the driving laser sources and in the approaches for the generation and detection of HHG and isolated attosecond pulses. Furthermore, a strong effort is needed for the interpretation of these very complex experiments. This special issue is devoted to the most recent and intriguing developments in these attosecond technologies.

The first crucial aspect that is addressed here is the next generation driving sources for strong field applications [33-37]. New approaches for high order harmonic generation and detection [38, 39], recent developments in the generation and characterization of attosecond pulses [40, 41] and the emerging role of FELs in the attosecond framework $[42,43]$ are also presented.

A large section of the special issue is finally devoted to the discussion of innovative approaches for studying attosecond dynamics [44-56].

\section{ORCID iDs}

Guest editorsJohan Mauritsson (1) https://orcid.org/0000-0002-7289-5072

Giulio Vampa (1) https://orcid.org/0000-0001-8492-3408

Caterina Vozzi i https://orcid.org/0000-0002-0212-0191

\section{References}

[1] Corkum P B and Krausz F 2007 Attosecond science Nat. Phys. 3 381-7

[2] Paul P M et al 2001 Observation of a train of attosecond pulses from high harmonic generation Science 292 1689-92

[3] Hentschel M et al 2001 Attosecond metrology Nature 414 509-13

[4] Drescher M et al 2002 Time-resolved atomic inner-shell spectroscopy Nature 419 803-7

[5] Wang $\mathrm{H}$ et al 2010 Attosecond time-resolved autoionization of argon Phys. Rev. Lett. 105 143002

[6] Goulielmakis E et al 2010 Real-time observation of valence electron motion Nature 466 739-43

[7] Eckle P, Pfeiffer A N, Cirelli C, Staudte A, Dörner R, Muller H G, Büttiker M and Keller U 2008 Attosecond ionization and tunneling delay time measurements in helium Science 322 1525-9

[8] Kraus P M et al 2015 Measurement and laser control of attosecond charge migration in ionized iodoacetylene Science $\mathbf{3 5 0} 790-5$ 
[9] Haessler S et al 2011 Self-probing of molecules with high harmonic generation J. Phys. B: At. Mol. Opt. Phys. 44203001

[10] Beck A R et al 2015 Probing ultrafast dynamics with attosecond transient absorption Chem. Phys. Lett. 624 119-30

[11] Calegari F et al 2014 Ultrafast electron dynamics in phenylalanine initiated by attosecond pulses Science 346 336-9

[12] Corkum P B 1993 Plasma perspective on strong field multiphoton ionization Phys. Rev. Lett. 711994

[13] Kulander K C et al 1993 Dynamics of short-pulse excitation, ionization and harmonic conversion Super-Intense Laser-Atom Physics (NATO ASI Series B) ed B Piraux, A L'Huillier and K Rzcazewski (New York: Plenum Press) p 95

[14] Antoine P et al 1996 Attosecond pulse trains using high-order harmonics Phys. Rev. Lett. 771234

[15] Kienberger R et al 2004 Atomic transient recorder Nature 427 817-21

[16] Sansone G et al 2006 Isolated single-cycle attosecond pulses Science 314 443-6

[17] Feng X X et al 2009 Generation of isolated attosecond pulses with 20 to 28 femtosecond lasers Phys. Rev. Lett. 103183901

[18] Brabec T and Krausz F 2000 Intense few-cycle laser fields: frontiers of nonlinear optics Rev. Mod. Phys. 72 545-91

[19] Gaumnitz T et al 2017 Streaking of 43-attosecond soft-x-ray pulses generated by a passively CEP-stable mid-infrared driver Opt. Express 25 27506-18

[20] Li J et al 2017 53-attosecond X-ray pulses reach the carbon K-edge Nat. Comm. 8186

[21] Cousin S L et al 2017 Attosecond streaking in the water window: a new regime of attosecond pulse characterization Phys. Rev. X 7041030

[22] Kühn S et al 2017 The ELI-ALPS facility: the next generation of attosecond sources J. Phys. B: At. Mol. Opt. Phys. 50132002

[23] Fleischer A, Kfir O, Diskin T, Sidorenko P and Cohen O 2014 Spin angular momentum and tunable polarization in high-harmonic generation Nat. Photon. 8 543-9

[24] Ferré A et al 2014 A table-top ultrashort light source in the extreme ultraviolet for circular dichroism experiments Nat. Photon. 9 93-8

[25] Schultze M et al 2014 Attosecond band-gap dynamics in silicon Science 346 1348-52

[26] Schlaepfer F et al 2018 Attosecond optical-field-enhanced carrier injection into the GaAs conduction band Nat. Phys. 14 560-4

[27] Zürch M et al 2017 Direct and simultaneous observation of ultrafast electron and hole dynamics in germanium Nat. Comm. 815734

[28] Moulet A et al 2017 Soft x-ray excitonics Science 357 1134-8

[29] Schiffrin A et al 2013 Optical-field-induced current in dielectrics Nature 49370

[30] Ghimire S et al 2011 Observation of high-order harmonic generation in a bulk crystal Nat. Phys. 7138

[31] Luu T T et al 2015 Extreme ultraviolet high-harmonic spectroscopy of solids Nature $\mathbf{5 2 1} 498$

[32] Huang S et al 2017 Generating single-spike hard x-ray pulses with nonlinear bunch compression in free-electron lasers Phys. Rev. Lett. 119154801

[33] Hammond T J 2017 Spatio-temporal coupling to create sub-femtosecond pulses J. Opt. 19 114002

[34] Bashinov A V et al 2017 Towards attosecond-scale highly directed GeV gamma-ray sources with multipetawatt-class lasers J. Opt. 19114012

[35] Ishii N et al 2018 Generation of sub-two-cycle millijoule infrared pulses in an optical parametric chirped-pulse amplifier and their application to soft x-ray absorption spectroscopy with high-flux high harmonics J. Opt. 20014003

[36] Harth A et al 2018 Compact $200 \mathrm{kHz}$ HHG source driven by a few-cycle OPCPA J. Opt. 20 014007

[37] Witting T et al 2018 Spatio-temporal characterisation of a $100 \mathrm{kHz} 24 \mathrm{~W}$ sub-3-cycle NOPCPA laser system J. Opt. 20044003

[38] Neyra E et al 2018 High-order harmonic generation driven by inhomogeneous plasmonics fields spatially bounded: influence on the cut-off law J. Opt. 20034002

[39] Högner M et al 2018 Tailoring the transverse mode of a high-finesse optical resonator with stepped mirrors J. Opt. 20024003

[40] Kovács K et al 2017 Attosecond lighthouse above $100 \mathrm{eV}$ from high-harmonic generation of mid-infrared pulses J. Opt. 19104003

[41] Ren X et al 2017 Single-shot carrier-envelope-phase tagging using an f-2f interferometer and a phase meter: a comparison J. Opt. 19124017

[42] Serkez S et al 2018 Overview of options for generating high-brightness attosecond x-ray pulses at free-electron lasers and applications at the European XFEL J. Opt. 20024005

[43] Finetti P et al 2017 Optical setup for two-colour experiments at the low density matter beamline of FERMI J. Opt. 19114010

[44] Vacher M et al 2017 Transition dynamics in two-photon ionisation J. Opt. 19114011

[45] Bandrauk A D et al 2017 Circularly polarized attosecond pulse generation and applications to ultrafast magnetism J. Opt. 19124016 
[46] Loriot V et al 2017 Angularly resolved RABBITT using a second harmonic pulse J. Opt. 19 114003

[47] Chovancova M et al 2017 Spatial transport of electron quantum states with strong attosecond pulses J. Opt. 19114008

[48] Zhao X et al 2017 A new method for accurate retrieval of atomic dipole phase or photoionization group delay in attosecond photoelectron streaking experiments J. Opt. 19 114009

[49] Ngoko Djiokap J M and Starace A F 2017 Doubly-excited state effects on two-photon double ionization of helium by time-delayed, oppositely circularly-polarized attosecond pulses J. Opt. 19124003

[50] Hoff D et al 2017 Using the focal phase to control attosecond processes J. Opt. 19124007

[51] Guichard R et al 2017 Compensation of high order harmonic long quantum-path attosecond chirp J. Opt. 19124011

[52] Liu Q et al 2018 Attosecond streaking metrology with isolated nanotargets J. Opt. 20024002

[53] Jordan I and Wörner H J 2018 Extracting attosecond delays from spectrally overlapping interferograms J. Opt. 20024013

[54] Dahlström J M et al 2017 Attosecond transient absorption of a bound wave packet coupled to a smooth continuum J. Opt. 19114004

[55] McDonald C R et al 2017 Strong optical field ionisation of solids J. Opt. 19114005

[56] Tzallas P et al 2018 Time gated ion microscopy of light-atom interactions J. Opt. 20024018 